\title{
CONSERVATIVE APPROACH FOR RECOVERING FROM PAIN AND STIFF ELBOW FOLLOWING TRICEPS TENDON AVULSION AND REPAIR
}

Thiruvarangan Suwaminathan *1, Niroshan Jayarathne ${ }^{2}$, Sukunan Gunasingam ${ }^{3}$.

${ }^{* 1}$ Clinical Physiotherapist, Department of Physical Medicine, Base Hospital, Kaluwanchikudy, Sri Lanka.

${ }^{2}$ Consultant Orthopaedic Surgeon, Teaching Hospital Batticaloa, Sri Lanka.

${ }^{3}$ Medical Superintendent, Base Hospital, Kaluwanchikudy, Batticaloa, Sri Lanka.

\section{ABSTRACT}

Purpose: The triceps tendon avulsion is a uncommon injury type among other orthopedic injuries, therefore availability of literature is very rare in related to triceps rupture injury and subsequent medical management and discussing the pathophysiology to recover normal functional capability.

Case presentation: A 32-year-old young man who had a history of accidental falls while doing farming activities and consequently had an avulsion of the left triceps and after five days from having had a fall, he underwent repair of the left triceps by the orthopedic surgeon. After three weeks following surgery, he was referred to physiotherapy. He complained of pain during elbow bending and straightening and was also more concerned about his reduced left elbow joint movement than previously.

Discussion: The patient underwent six weeks' rehabilitative physical therapy interventions by carefully examining the elbow joint motion limitations in relation to injury type and joint stiffness.

Conclusion: The purpose of this case report was to present the choosing of a course of physiotherapy interventions based on biomechanical analysis of elbow movements in order to return to regular activities of daily life effectively after undergoing triceps repair associated with pain and stiffness.

KEY WORDS: Triceps Avulsion, Elbow Stiffness, Rehabilitation.

Address for correspondence: Thiruvarangan Suwaminathan, Clinical Physiotherapist, Department of Physical Medicine, Base Hospital, Kaluwanchikudy, Sri Lanka. E-Mail: t.ranga13@gmail.com

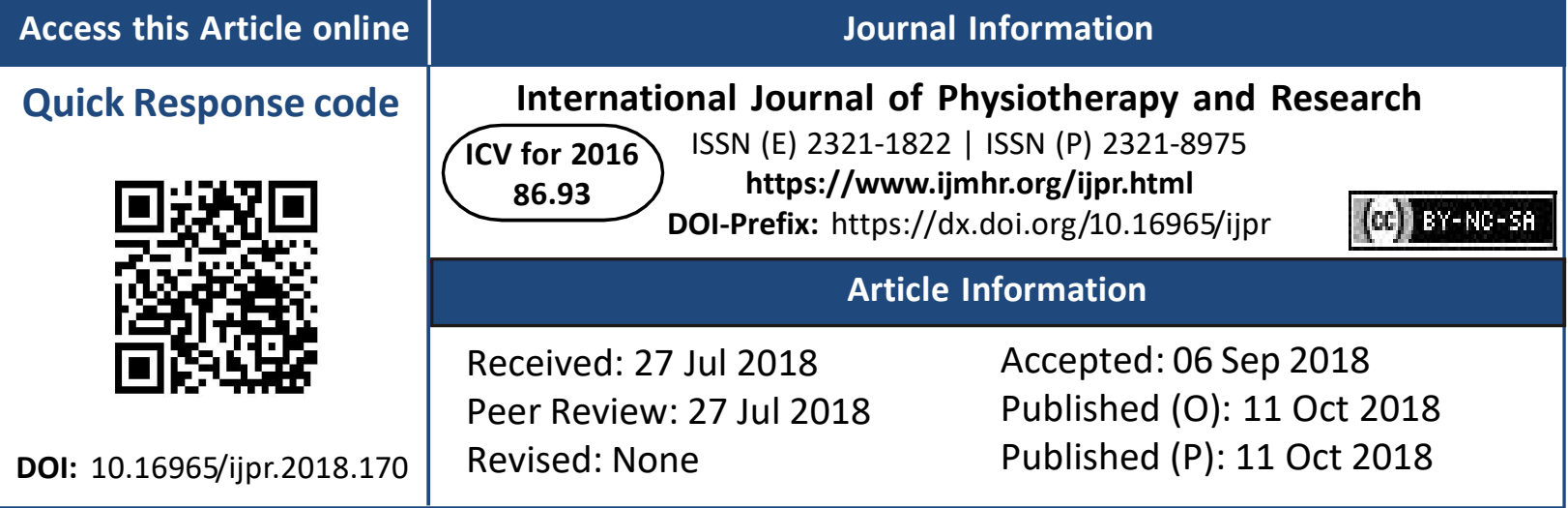

\section{INTRODUCTION}

Avulsion of the triceps tendon is a very rare injury and it represents less than $1 \%$ of all tendon injuries in the upper limb [1]. Triceps tendon ruptures were reported in the literature in approximately $75 \%$ of male patients, with a male-to-female ratio of $3: 1$ and the mean age at injury was around 26 years [2]. The susceptibility of the elbow joint to develop stiffness after even mild trauma is common and can cause sizeable impairment of upper limb functions. There are many factors involved in elbow joint stiffness and loss of movement. The main factors are the complexity of the joint surfaces and the high degree of soft tissue damage by trauma. In addition to that, elbow joint stiffness can be brought by poor rehabilitation and unnecessarily prolonged immobilization [3]. 
stiffness is dependent on meticulous clinical evaluation to determine the anatomical changes and subsequent ongoing pathological process in order to design an appropriate treatment regimen thus physiotherapists can have a major role to play in overcoming the problems related to loss of range of motion subsequently reducing upper limb functional activities.

\section{CASE PRESENTATION}

Subjective examination: A case report demonstrated a 32-year-old young man who had a history of accidental fall while carrying out farming activities and consequently had an avulsion of the left triceps and after five days having had a fall, he underwent repair of the left triceps by the orthopedic surgeon. After three weeks, he was referred to physiotherapy. He complained about the pain from the anteromedial aspect and posterior surface of the left elbow joint and was more concerned about reduction in his left elbow joint movement which interrupted his ability to carry out his occupation and also caused to weakening of his mental capabilities. The pain was sharp on left elbow bending and he experienced dull pain at night which disturbed his normal sleeping habits. The pain was eased to a certain extent by medication. He had no history of comorbidities.

Physical Examination: The left elbow examination was accomplished by a systemic approach of look, feel and move. It contains inspection, palpation, the range of motion, strength testing and neurological assessment. ${ }^{[4]}$ The initial assessment looked at plumb line standing anterior to a posterior view, left side cubits valgus deformity was noticed accompanying with an increase in the carrying angle and also slight swelling surrounding the joint area. Then, soft tissue inspection was carried out on the left elbow joint. His left anteromedial and posteriorlateral joint margin along with the head of the radius were tender under palpation and the distal part of the left triceps muscle near to the tenomuscular junction exhibited severe tenderness upon palpation. Next, left elbow joint range of motion was examined. The motion arc was less than 50 . Flexion was mostly diminished only about 30 o flexion and the terminal elbow extension was limited to around 20․ Supination and pronation were also limited into their end ranges. The passive motion was performed to make a differential diagnosis between neuromuscular and articular pathology. The left elbow joint all directions PROM were relatively equal to AROM.

The joint play movement restriction pattern was identified and classified according to Kay classification as type II. He described classification for elbow stiffness, based on the components involved in the process. In type I, there would only be isolated contracture of soft tissues. In type II, there would be contracture of soft tissues associated with heterotopic ossification. ${ }^{[5]}$ In type III, there would be contracture of soft tissues associated with a consolidated joint fracture, without dislocation. In type IV, the contracture of soft tissues would be associated with the defective consolidation of the joint fracture. In type $V$, a cross-joint bone bar would be present. ${ }^{[6]}$ Pain and soft tissue tightness were the limiting factors for the diminished range of motion of the left elbow joint. MMT revealed weakness in all left elbow functional movements. Pain and functional assessment were measured by VAS and Mayo elbow scoring system respectively. ${ }^{[7]}$ Performing provocative neurological tests were within normal limits to rule out the presence of symptoms relating to the ulnar nerve, moreover the left shoulder and wrist joint movements and functional capabilities were normal.

Fig. 1: Series of plain lateral view radiographs of the left elbow joint.
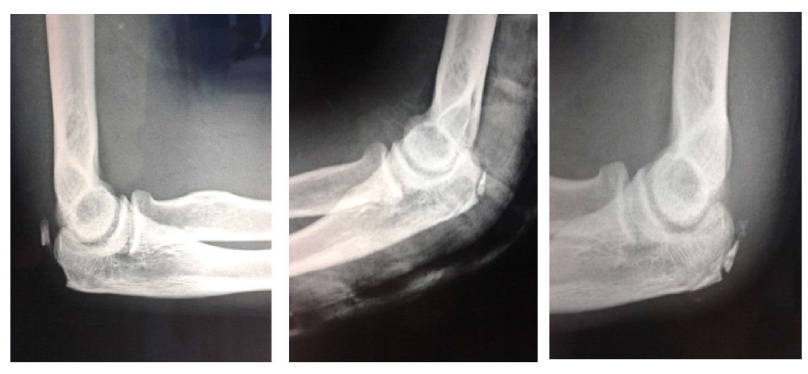

Investigation: A series of plain lateral view radiographs of the left elbow joint were taken. Figure:1(A), Lateral X-rays of the affected elbow showed a small piece of avulsed osseous fragment from the olecranon ('flake' sign) close to the tip of the olecranon, Figure:1(B) immediately after undergoing triceps repair with posterior back slap and Figure:1(C) after 6 weeks 
of physiotherapy rehabilitation [8].

\section{TREATMENT}

Surgical Intervention: The patient had a ruptured left distal triceps tendon and consequently, the repair of the triceps tendon was performed on the fifth day after injury by an orthopedic surgeon. The triceps tendon was found to be retracted by $2 \mathrm{~cm}$ proximal to the tip of the olecranon. The edges of the tendon were cleared and were released from the surrounding tissues. The triceps tendon was repaired using the Krakow method, constituting a 4-strand suture. The suture was used with No.2 Ethibond. The triceps tendon stump was positioned at the original insertion into the olecranon to restore the excursion length of the tendon by passing the sutures through bone tunnels made into the olecranon. Postoperatively, the elbow was placed in a posterior splint at 20 의 flexion for two weeks [9].

Fig. 2: Krakow stitch for repairing the ruptured distal triceps tendon,

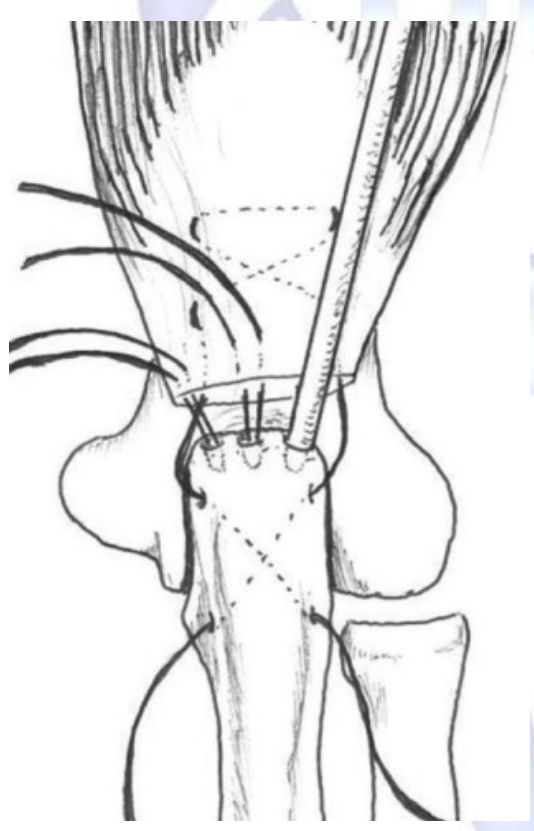

Physical Therapy Intervention: After 2 weeks, the posterior splint was removed and the patient was referred to physical therapy for progressive elbow flexion, however the surgeon advised $0-90^{\circ}$ limited flexion for a further two weeks. The patient was seen three times per week for two weeks. At the end of 4 weeks, the orthopaedic surgeon encouraged past elbow flexion of more than 900 and achieves full range of extension. At the end of 6weeks, active flexion and extension against gravity were allowed but a forced extension was prohibited for an additional 4 weeks. After 12 weeks, the patient was allowed to do gradual resistance exercises [9]. Stiffness is a frequent complication after elbow trauma, and the recovery of a functional arc of mobility may be difûcult. The intensity of elbow stiffness following the trauma is varying; therefore therapeutic intervention is determined by the individual patient's subjective complaints and the objective evaluations [10]. The majority of patients have elbow range of motion restriction and these hinder their upper limb functional activities due to poor intervention approach [3]. This problem can be overcome by an individualized physical therapy regimen based on the individual's comprehensive clinical evaluation. It includes passive and active joint mobilization, soft tissue mobilization, muscle stretching, joint play manipulation and muscle strengthening. Physical therapists have advocated the use of joint play manipulation, which is considered to be an effective treatment for enhancing ROM in the patient with post-trauma stiffness of the elbow [11].

Outcomes: After 8 weeks of physiotherapy interventions, the VAS and Mayo elbow score was improved from $8 / 10$ to $2 / 10$ and from 30 to 85 respectively. The left elbow joint all ROM were achieved normally. The patient could perform all his functional activities of daily living mostly pain-free.

\section{DISCUSSION}

Anatomy: There are actually three joints at the elbow. The first being the hinge joint formed between the distal humerus and the proximal ulna called the humeroulnar joint, which allows flexion and extension at the elbow. The second is the humeroradial joint between the distal humerus and proximal radius which again allows flexion and extension but is also involved in the motion of the forearm supination and pronation. The third is a pivot joint formed between the proximal radius and ulna and is called the proximal radioulnar joint. As all synovial joints, the elbow joint has a capsule enclosing the joint. This in itself is strong and fibrous, strengthening the joint [12]. Moreover, this joint capsule is strengthened by ligaments medially and laterally. There are three main ligaments supporting 
the elbow joints; medially the ulnar collateral ligament, which consists of two triangular bands, anterior and posterior both bands arise from the medial epicondyle and pass over the inside of the elbow joint. The anterior portion then attaches to the front part of the top of the ulna, known as the coronoid process, and the posterior part to the back of the olecranon process of ulna. Laterally, the radial collateral ligament is a short, narrow band which passes from the base of the lateral epicondyle to the annular ligament. The annular ligament is a band of fibres which circle the head of the radius, maintaining contact between the radius and humerus. Also the elbow joint has a bursa which is a membranous sac filled with synovial fluid. It acts as a cushion for the moving parts of a joint and subsequently prevents degenerative damage [13].

Pathophysiology: The elbow is particularly prone to stiffness following traumatic injury. This propensity has been attributed to several factors, including the congruous nature of the joint, the presence of three articulations within a synovial lined cavity, and the close relationship of the joint capsule to the intracapsular ligaments and surrounding muscles. Furthermore, the elbow capsule itself plays a role in arthrofibrosis. Gallay et.al found the stiff elbow capsule to be one-sixth as compliant as that of the normal elbow, concluding that it is altered structurally and biomechanically. They hypothesized that these changes involved hypertrophy of the capsule or a change in its collagen content [14].

Experimental laboratory-based studies have investigated the biochemical and biological alterations that occur in periarticular tissues in response to trauma. Cohen et al reported that stiff elbows presented a thin capsule with a disorganized collagen matrix, increased inflammatory cytokine levels, and fibroblastic infiltration, thus characterizing a fibrotic and inflammatory condition [15].

Other authors have documented increases in the formation of cross-linked collagen, associated with decreased proteoglycan content and water in joints presenting contracture, along with changes to the regulation of growth factor. In addition to that, an indirect relationship between trauma and elbow stiffness due to unnecessary prolonged immobilization and pitiable rehabilitation.

\section{CONCLUSION}

The stiffness of the elbow is very common even after mild trauma and can be associated with significant functional impairments. The rehabilitation of the post-traumatic stiff elbow must follow a comprehensive approach by analyzing of the intensity of trauma; meticulous physical examination is related to biomechanical aspect and limited ROM, because many studies have demonstrated that muscle and joint soft tissues both contribute to the total joint stiffness. Therefore, the therapist must treat all aspects of joint stiffness. It is important to incorporate joint mobilization, appropriate stretching techniques and strengthening exercises into the effective rehabilitation regimen of a post-traumatic stiff elbow. Physiotherapy interventions will remain to have a vital role in treating patients who have trauma in their elbow in order to encounter the possibility of developing stiffness and functional limitations to have free functional capability in the day to day livings and recreational life.

\section{ABBREVIATIONS}

VAS - Visual Analog Scale

AROM - Active Range of Motion

PROM- Passive Range of motion

MMT - Manual Muscle Testing

\section{ACKNOWLEDGEMENTS}

The authors wish to thank Mr. Mark Williams, Senior Lecturer, British Way English Academy who provided the proofreading services in the final stage of the manuscript submission process in regard to gain the potential language level.

\section{Conflicts of interest: None}

\section{REFERENCES}

[1]. Anzel SH, Covey KW, Weiner AD, Lipscomb PR. Disruption of muscle and tendons. An alaysis of 1014 cases. Surgery. 1959;45(3):406-14.

[2]. Bach BR Jr, Warren RF, Wickiewicz TL .Triceps rupture. A case report and literature review. Am J Sports Med. 1987;15: 285-289.

[3]. Morrey BF. The posttraumatic stiff elbow. Clin Orthop Relat Res. 2005;431:26-35. 
[4]. Gareth TJ, Kathrin M, Gary JM, Keith TP, David C, Karen WB, et al. Maintained physical activity and physiotherapy in the management of distal upper limb pain - a protocol for a randomised controlled trial Musculoskeletal Disorders. 2014;15:71.

[5]. Colon H. Wilson. Clinical Methods: The History, Physical, and Laboratory Examinations. 3rd edition chapter 164.

[6]. Kay NR. Arthrolysis of the posttraumatic stiff elbow. In: Stanley D, Kay NR, editors. Surgery of the elbow: practical and scientific aspects. London: Edward Arnold; 1998. p. 228-34.

[7]. Derya Celik. Psychometric properties of the Mayo Elbow Performance Score. Rheumatology International. 2014;35(6).

[8]. Cyriax J. Diagnosis of Soft Tissue Lesions. Baltimore: Williams and Wilkins, Textbook of Orthopaedic Medicine. 1975;180-228.

[9]. Sharma P, Vijayargiya M, Tandon S, Gaur S. Triceps tendon avulsion: a rare injury. Ethiop J Health Sci. 2014;24: 97-99.
[10]. Nirschi RP, Morrey BF. Rehabilitation. In:Morrey $B F$,ed. The elbow and its disorders. $2^{\text {nd }}$ edition. Philadelphia:WB Saunders, 1993:173-80.

[11]. Hickey CJ. Arm wrestling with isokinetic gender differences: future implications for rehabilitation, recovery, and success. Journal of orthopaedic \& sports physical therapy. 2014; 44(1):83.

[12]. Warwick R. Williams P. Gray's Anatomy, 35th British Ed. Philadelphia: WB Saunders Co, 1973, 399407.

[13]. Stefan F, Ranjan G, Thay QL. Anatomy and Biomechanics of the Elbow Joint. Techniques in Hand and Upper Extremity Surgery. 2003; 7(4):168-178.

[14]. Gallay SH, Richards RR, O'Driscoll SW. Intraarticular capacity and compliance of stiff and normal elbows. Arthroscopy.1993;9(1):9-13.

[15]. Cohen MS, Schimmel DR, Masuda K, Hastings $H$, Muehleman C. Structural and biochemical evaluation of the elbow capsule after trauma. J Shoulder and Elbow Surg. 2007;16(4):484-490.

How to cite this article:

Thiruvarangan Suwaminathan, Niroshan Jayarathne, Sukunan Gunasingam. CONSERVATIVE APPROACH FOR RECOVERING FROM PAIN AND STIFF ELBOW FOLLOWING TRICEPS TENDON AVULSION AND REPAIR. Int J Physiother Res 2018;6(5):2886-2890. DOI: 10.16965/ijpr.2018.170 